performed, and 20 patients underwent a multi-faceted procedure. $56 \%$ of patients had a BMI above the normal range. Median length of stay was 8 days, IQR 7-10, range 3-42.

68 complications occurred in 46 patients. There were 2 deaths. There were 54 complications requiring intervention, and 14 self-limiting complications. 28 patients had 1 complication, 14 patients had 2 complications and 4 patients had 3 complications. The most common complications requiring intervention were post-operative chest infection requiring oral or IV antibiotics $(n=19)$, atrial arrhythmia requiring antiarrhythmic therapy or cardioversion $(n=7)$, post-operative wound infection requiring IV or oral antibiotics $(n=7)$, and pericardial effusion requiring drainage $(n=5)$. Self-limiting complications included 1 patient with nodal rhythm with pauses, 2 patients with an acute confusional state, 1 patient with a groin haematoma (following fem-fem bypass), 1 patient with a residual VSD following resection of double chambered right ventricle and 1 patient with gastroenteritis.

Apart from the 2 deaths, there were 4 patients who had long term sequelae from their complication. 1 patient in their 60 's suffered a peri-operative cerebellar stroke, 1 patient required a permanent pacemaker, and 2 patients had vocal cord palsy leading to hoarse voice.

Post-operative complications increased in incidence with both increasing age and increasing BMI. (Figs 1\&2)

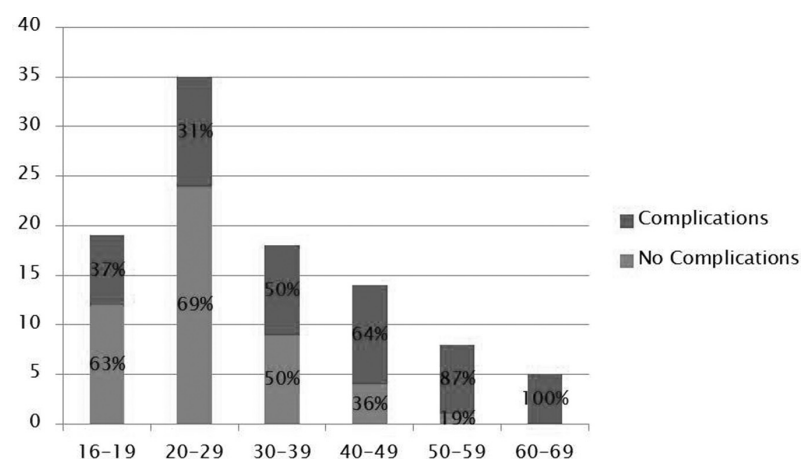

Abstract 85 Figure 1 The incidence of post-operative complications according to patient age.

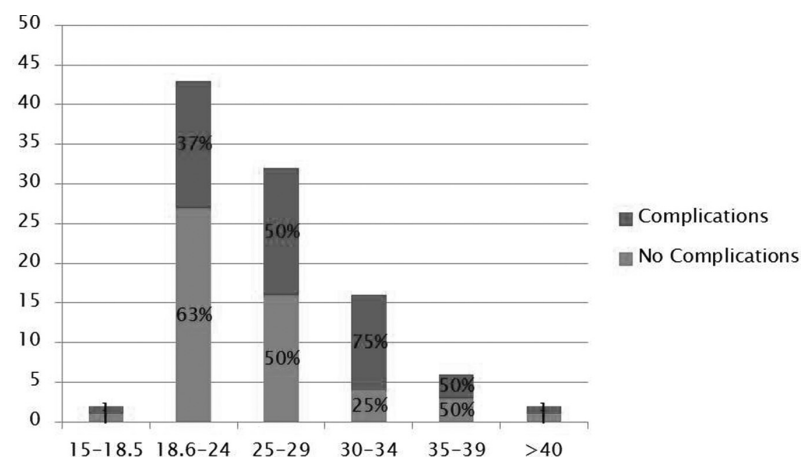

Abstract 85 Figure 2 The incidence of post-operative complications according to patient BMI.
Conclusions Increasing numbers of cardiac surgical procedures are being performed in ACHD patients because of their increasing numbers and complexity. This study demonstrates that even complex surgery can be undertaken at low mortality risk. Just under $50 \%$ of patients experience one or more perioperative complications but the vast majority are successfully treated with no long term adverse consequences. Complications appear to increase with advancing age and BMI.

\section{Stable IHD/Prevention/Hypertension/Lipids}

\section{ACHIEVEMENT OF BLOOD PRESSURE, LIPIDS AND GLUCOSE TARGETS IN PATIENTS WITH CORONARY DISEASE OR AT HIGH RISK OF DEVELOPING ATHEROSCLEROTIC CARDIOVASCULAR DISEASE IN EUROPE: RESULTS FROM EUROASPIRE SURVEY}

${ }^{1}$ Kornelia Kotseva*, ${ }^{1}$ David Wood, ${ }^{2}$ Catriona Jennings, ${ }^{3}$ Dirk De Bacquer, ${ }^{3}$ Guy De Backer. ${ }^{1}$ Imperial College London; ${ }^{2}$ Imerial College London; ${ }^{3}$ University of Ghent

\subsection{6/heartjnl-2017-311726.85}

Introduction EUROASPIRE IV is a cross-sectional survey undertaken in 26 European countries to evaluate the implementation of the Joint European Societies guidelines on cardiovascular (CVD) prevention in everyday clinical practice.

Methods Patients $<80$ years with 1 ) coronary disease or 2) identified as high CVD risk on the basis of being on blood pressure and/or lipid-lowering medication and/or having diabetes, were interviewed at least six months after the recruiting coronary event or the start of medication.using standardised methods and instruments.

Results A total of 7998 coronary patients (24\% females) and 4579 high CVD risk individuals $(59 \%$ women) were interviewed. The risk factor control was poor in both groups. Among those on blood pressure lowering medication the target of $<140 / 90 / 90 \mathrm{~mm} \mathrm{Hg}(<140 / 80 / 80 \mathrm{~mm} \mathrm{Hg}$ in people with diabetes) was achieved by $53 \%$ of coronary patients and $43 \%$ of high CVD risk individuals. Only $21 \%$ of coronary patients attained the LDL-cholesterol goal of $<1.8 \mathrm{mmol} / \mathrm{L}$ and $33 \%$ of high CVD risk individuals achieved the target of $<2.5 \mathrm{mmol} / \mathrm{L}$. Among people treated for type 2 diabetes mellitus, $53 \%$ of coronary patients and $59 \%$ of high CVD risk individuals achieved the glycated haemoglobin (HbA1c) target of $<7.0 \%$. Compared with data from previous surveys the control of blood pressure and LDL-cholesterol in coronary patients improved significantly. There was a trend in improving of blood pressure and lipids management in patients at high CVD risk. In both groups, glycaemic control in patients with previously known diabetes was similar to that observed in the third survey.

Conclusion Despite advances in patient management there is further potential to improve risk factor control in patients with coronary disease and those at high risk of developing CVD. Although the risk factors studied were better controlled than in previous surveys, there is still considerable potential to further reduce cardiovascular risk through lifestyle and optimised cardioprotective medications. A significant gap continues to exist between the prevention guidelines and their adherence in everyday clinical practice in Europe. 